\title{
QUANTUM-RELATIVISTIC PHYSIS
}

\author{
ENRICO R.A.C. GIANNETTO (*)
}

SUNTO. - Si analizza la nascita della scienza moderna come "nuova filosofia": si tratta di una specifica forma di "filosofia pratica", nata come conseguenza della rivoluzione nominalista francescana e della Riforma. Questa analisi permette di comprendere le svolte implicite nell'ontologia e nella gnoseologia apportate dalla rivoluzione scientifica. Si compara lo sviluppo parallelo dell'ontologia e della gnoseologia nella filosofia successiva. Si discutono, poi, in questa luce, i problemi filosofici posti dalla fisica quantistica per la conoscibilità della Natura. In questa prospettiva, si delineano i ribaltamenti delle gerarchie disciplinari filosofico-scientifiche e della metafisica soggettivistica e antropocentrica occidentale, implicati dalla rivoluzione quantistica.

$* * *$

ABSTRACT. - Physics is considered as a particular kind of natural philosophy, related to a gnoseology of experimental actions. Quantum-relativistic physics implies the emergence of an indeterministic, entangled Nature, which shows itself as a dynamically organized totality like ancient Physis, in which mankind can understand itself in its existential and ethical dimension.

\section{INTRODUZIONE}

Il tema "Realtà senza realismo" pone implicitamente il problema della filosofia "veicolata" dalla fisica o dell'interpretazione filosofica della fisica, in relazione alla questione della "realtà". Vi è la necessità di una diretta conoscenza storica e teoretica della fisica coinvolta per potersi orientare all'interno della varietà delle possibili interpretazioni, e di una diretta conoscenza storica e teoretica della filosofia per poterne valutare le conseguenze per la comprensione della questione della "realtà fisica". Si deve poi tenere conto dei vari sviluppi della filosofia della scienza che spe-

(*) Università degli Studi di Bergamo, Italia. E-mail: enrico.giannetto@unibg.it 
cificamente si è rivolta alla comprensione di tali problematiche, con l'avvertenza, però, che non solo questa, in gran parte, non soddisfa i requisiti sopra menzionati di una conoscenza diretta della fisica e della filosofia, ma anche si basa già su una semplificazione storiografica ed epistemologica di una situazione, che, come si vedrà, è ben più complessa: si basa sulla considerazione della scienza contemporanea come del tutto separata e separabile dalla filosofia, e viceversa della filosofia come del tutto separata e separabile, nella sua storia e nella sua costituzione, da quella filosofia della Natura pratico-sperimentale che è la scienza moderna.

D'altra parte, se, per differenziarle, si mettesse l'accento su delle mere pratiche matematiche e tecnico-sperimentali della scienza, si dovrebbe arrivare alla conclusione, pure erronea, che non esiste un "pensiero scientifico", perché, come diceva Martin Heidegger, si dovrebbe dire che la scienza calcola e opera ma non pensa[1, pag.88][2, pagg. 41 e 116].

\section{LA NASCITA E LA NATURA DELLA SCIENZA MODERNA}

Bisogna, invece, ricomprendere la scienza come una forma di pensiero che, attraverso modelli tecnici, si concretizza in azione, e quindi come una particolare forma di filosofia pratica che contesta la possibilità di concepire ancora la filosofia, secondo l'antico paradigma greco, come un'attività meramente contemplativa, che la qualifichi come una filosofia teoretica pura. Dopo la rivoluzione francescana in filosofia, operata soprattutto da John Duns Scotus (1265-1308) e William of Ockham (1287-1347), che destituì di fondamento teologico la filosofia teoretica greca della Natura [3], la filosofia della Natura si dovette ripensare in termini di una filosofia pratica. La questione è legata al fatto che la Natura non poteva più essere pensata come prodotto razionale di un intelletto divino interno od esterno ad essa, ma piuttosto doveva essere considerata come la creazione di un Dio cristiano che si caratterizza propriamente in termini di Volontà ovvero di Amore. L'imperscrutabilità della Volontà divina e l'incondizionatezza dell'Amore hanno come conseguenza l'assoluta contingenza della Natura e la sua inconoscibilità a priori da parte di una ragione teoretica pura. Il mondo naturale non poteva essere più considerato oggettivisticamente, secondo la prospettiva intellettualistica greca, ma, derivando dall'azione e dalla volontà della soggettività divina, presentava connota- 
zioni soggettive impredicibili. E ciò distruggeva anche la possibilità di una filosofia teoretica della Natura che non poteva che basarsi su una metafisica teologica.

Richiamandosi all'originaria caratterizzazione cristiana di Dio in termini di Amore come libera volontà, la prospettiva francescana si oppose alla concezione della divinità greca, come quella aristotelica, chiusa nella sua perfezione intellettuale di una autocontemplazione del pensiero puro, e distrusse la connessione medioevale del Cristianesimo con la teologia, la filosofia, la cosmologia e la fisica greche. Si trattò di un processo ancora più ampio di de-ellenizzazione del Cristianesimo, che causò la rottura del matrimonio del Cristianesimo con la filosofia greca, e, in particolare, con l'aristotelismo, e l'avvento di una nuova fase di radicalizzazione dello scontro del Cristianesimo con la cultura occidentale, che riproponeva quanto già avvenuto alle sue origini.

La questione teologica delle argomentazioni de potentia Dei absoluta come distinte da quelle de potentia Dei ordinata poneva, in effetti, il problema non solo dell'assoluta contingenza della Natura relativa a ciò che Dio avrebbe potuto fare e non ha fatto, ma anche della possibile esistenza di una parte della Natura, di cui non abbiamo o non possiamo avere esperienza (per esempio, l'esistenza del vuoto, l'esistenza di altri infiniti mondi), producibile dall'azione di Dio. Questa prospettiva teologica stimolò l'immaginazione scientifica a discutere non solo ciò che è oggetto di usuale esperienza in Natura, ma anche ciò che è producibile "artificialmente" in Natura ed è al di là dell'esperienza diretta umana.

Questa divergenza non ricomponibile fra ragione e fede, e quindi fra filosofia teoretica e fede, trovò il suo culmine nella prospettiva di Martin Luther (1483-1546) [4].

Con la Riforma, il distacco dalla contemplazione teoretica greca come ideale gnoseologico ed etico di perfezione modellato sulla divinità aristotelica quale noesis noeseos (come puro pensiero di pensiero) fu ulteriormente perseguito fino all'abolizione protestante delle forme di vita monacali. Come solo le opere nel mondo possono essere prova della Fede e della Grazia e così della Salvezza (e non la vita contemplativa monacale), così solo la producibilità e la riproducibilità effettiva dei fenomeni tramite le azioni sperimentali assunsero il ruolo di prova della comprensione della Natura (al posto della contemplazione teoretica della filosofia greca dominante).

La nuova filosofia naturale di William Gilbert, Thomas Harriot, di Galileo Galilei e di altri quali Blaise Pascal, fu una fisica sperimentale, 
basata su una gnoseologia dell'azione sperimentale che costituiva l'unica conoscenza possibile della Natura come a posteriori. La filosofia della Natura, avendo perso il suo fondamento teoretico nella metafisica teologica, trovò un nuovo fondamento in una prassi sperimentale in cui la tecnica perdeva la sua connotazione puramente strumentale per assumerne una conoscitiva: nella prassi sperimentale, il pensiero si concretizzava, si faceva azione potendosi così misurare effettivamente con il mondo: del pensiero puro non si può stabilire se sia in corrispondenza con la realtà, solo del pensiero che si fa azione concretizzandosi in modelli realizzati tecnicamente si può stabilire una corrispondenza con la Natura. Si passa così dall'assunzione teoretica di una verità staticamente attribuità a un pensiero astratto, ad un processo dinamico di verific-azione del pensiero nella realtà. Nell'azione sperimentale il rapporto tecnico con il mondo viene abbandonato perché le finalità della tecnica e degli strumenti vengono ribaltate: non sono più finalità di vantaggio umano, ma di comprensione della Natura; gli strumenti tecnici sono defunzionalizzati dai loro fini e trasformati in un'operatività che lascia emergere dal profondo e dal lontano, non tangibili, la Physis al di là dell'esperienza umana e delle sue finalità, in una manifestatività mediata dagli strumenti. La meccanica in Galileo viene così trasformata da tecnica in scienza fisica. L'essere della Natura non è più colto in termini della sua utilizzabilità e nei limiti del suo manifestarsi nelle dimensioni della nostra esperienza umana, ma piuttosto gli esperimenti rivelano, in una forma velata dagli strumenti, una nuova verità della Natura come aletheia nel senso di una sua non-celatezza, di un'essere della Natura indipendente dall'umanità e dalle finalità antropocentriche, che ci obbliga a ri-definire l'orizzonte di significatività in cui ricomprendere il senso dell'esistenza umana come essere-parte-della-Natura, di una Natura che trascende l'esperienza umana. Superare con gli esperimenti l'esperienza umana non è solo superarne i limiti e le ambiguità, ma più profondamente implica l'accessibilità di un nuovo senso intrinseco della Natura non-umano, oltre quello antropocentrico definibile nell'esperienza umana.

Il fenomeno naturale, così, per Galileo, non è più interpretabile nei termini del linguaggio umano, dei suoi sensi e dei suoi fini, ma piuttosto si deve interpretare nei termini del linguaggio divino che ha la perfezione della matematica, di un senso e di una finalità divina che oltrepassa l'umano: è l'essere delle cose come simbolo aritmetico-geometrico dell'attività di scrittura matematica del libro della Natura da parte di Dio, la cui interpretazione ne costituisce il senso proprio, rispetto al 
quale gli altri sensi sono derivati e fasulli; il senso di una cosa sta nella composizione matematica con le altre in un tutto fisico ordinato e intelligente. Quella di Galileo è comunque una geometria meccanica e un'analisi atomistica, cioè una matematica a posteriori rispetto alla fisica.

Tuttavia, la ricomprensione dell'umano all'interno della Natura, il senso esistenziale proprio del fenomeno della Natura nell'orizzonte della significatività aperto dalla nuova filosofia emerge pienamente solo nella fenomenologia fisica di Pascal; la simbolizzazione matematica delle relazioni istituibili fra i fenomeni in base alle azioni sperimentali si poteva considerare una mera tachigrafia di relazioni fisicamente performabili e comunque era a posteriori rispetto alla sperimentazione e costituiva una comprensione della Natura sempre incerta e probabilistica: l'esistenza del vuoto costituiva la cifra della manchevolezza ontologica della Natura, compresa la natura umana [5].

\section{LA METAFISICA TEOLOGICO-MATEMATICA NEWTONIANA E TECNICA}

La nuova filosofia naturale, dopo qualificata come "scienza moderna", non ebbe però quella continuità solitamente attribuita ad essa dai fisici e dagli storici. Certo, già da Francis Bacon, il rapporto fra scienza e tecnica è stato del tutto capovolto a favore della tecnica, o comunque è stato continuamente soggetto a ribaltamenti: la tecnica moderna non è meramente scienza applicata, ma, come aveva compreso Martin Heidegger, da Bacon in poi diventò anche il presupposto alla base della scienza moderna, della sua matematizzazione e del suo considerare l'essenza della Natura come qualcosa di quantificabile [4, capp. VII, VIII, IX]. Dal XVII secolo in poi la Natura è stata concepita come un mero oggetto di cui si può calcolare matematicamente il comportamento, e non come un soggetto vivente che esige il nostro rispetto da un punto di vista etico, solo per giustificare lo sfruttamento tecnico della Natura e degli altri esseri viventi da parte dell'uomo. La scienza moderna con la sua correlata teologia non diventò altro che l'ideologia della tecnica: questa finalità al termine del XIX secolo portò a presupporre la riduzione della Natura ad un fondo di risorse, di 'energie' quali 'capacità di compiere lavoro meccanico', a disposizione della 'volontà di potenza' dell'uomo.

Gli sviluppi della scienza del Novecento, che pure hanno messo in discussione la possibilità di matematizzare esattamente la Natura 
(primi fra tutti quelli della fisica quantistica con il suo "principio d'indeterminazione", che in generale non permette una descrizione esatta della Natura in termini di energia), sono stati comunque usati per creare nuovi dispositivi tecnici che permettono all'essere umano un dominio della Natura anche a livello microscopico.

Questa finalità tecnica di dominio della Natura diventò tutt'uno con una metafisica matematica [6]. L'istituzione di "principi della filosofia" naturale stabiliti teoreticamente dall'intelletto da Cartesio come generali "leggi di Natura" a priori ristabilì la priorità greca del pensiero puro. Questa impostazione fu ripresa da Newton con l'assunzione a priori di "principi matematici della filosofia naturale" e riportò la fisica a dipendere da una metafisica teologica che si traduceva in una metafisica matematica: Newton aveva postulato teologicamente l'esistenza di uno spazio e di un tempo matematici assoluti quali attributi di Dio pur di poter fondare teoreticamente la verità del sistema del mondo copernicano-kepleriano che richiedeva lo stabilimento di un moto assoluto della Terra intorno al Sole.

Dopo la rivoluzione scientifica, era sorta l'epistemologia come disciplina autonoma e separata, quale teoria della conoscenza scientifica, all'interno della gnoseologia come teoria della conoscenza: l'epistemologia apparentemente si staccava e si rendeva autonoma dalla metafisica e dall'ontologia che avevano avuto il ruolo di "scienze prime", di fondamento alle altre, nel pensiero antico e in quello medioevale insieme alla teologia: con la "rivoluzione scientifica", con Descartes e, definitivamente, con Kant, l'epistemologia si veniva ad affermare come "scienza prima", di fondamento a tutte le altre. Era la teoria della conoscenza scientifica (epistemologia), edificata sul principio di causalità (efficiente, meccanica), sul metodo assiomatico-deduttivo della matematica (sul modello della geometria euclidea) e sul metodo sperimentale come verifica di quello, a determinare ciò che è realmente, e non era, viceversa, la teoria della conoscenza a dipendere dalla struttura della realtà, da ciò che è (ontologia).

\section{LA RIVOLUZIONE QUANTO-RELATIVISTICA}

Questa metafisica teologico-matematica ed epistemologica fu dominante per un paio di secoli e fu scardinata dall'opera di Henri Poincaré che si rifaceva a Leibniz che già aveva contestato le conclusio- 
ni di Newton sia sul piano teologico che sperimentale per l'inosservabilità dello spazio e del moto assoluti [7]. Bisognava riconoscere sperimentalmente la relatività generale del moto, la relatività dello spazio e del tempo, la convenzionalità-relatività della scrittura matematico-geometrica, la "convenzionalità" del tutto ipotetica delle leggi di Natura quali principi della fisica che possono essere considerati solo come generalizzazioni induttive degli esperimenti mai teoreticamente fondabili. Ma c'è di più: la fine della materia come sostanza permanente nel tempo, come noumeno di cui i fenomeni fisici sono la manifestazione. Il fenomeno naturale è l'essere delle cose che è simbolo dell'attività dinamica stessa della Natura nella sua totalità di attuazione, del campo elettromagnetico come luce visibile e invisibile, quale principio fisico attivo di cui la materia-sostanza è solo manifestazione secondaria apparente: il fenomeno naturale è luce non-nascosta che si automanifesta negli esperimenti. Da qui, la nuova dinamica relativistica, poi portata a compimento da Einstein, la nuova crono-geometria quadridimensionale spazio-temporale introdotta perché le onde elettromagnetiche sono definibili solo come funzioni spazio-temporali, la riconduzione della materia a energia del campo e dell'essere delle cose a insiemi di eventi spazio-temporali legati alla propagazione spazio-temporale delle onde elettromagnetiche.

La matematica tornava a essere determinata, e invero stravolta da geometria in crono-geometria non-euclidea, a posteriori rispetto alla fisica sperimentale: la metafisica teologico-matematica di Newton fu così almeno parzialmente dissolta [4, capp. XIII, XIV].

Tuttavia, la decostruzione definitiva della metafisica teologicomatematica che costituiva la cosidetta "fisica classica" si attuò solo con la rivoluzione quantistica [4, cap. XV].

Heisenberg, nel 1925, aprì la strada alla meccanica delle matrici, richiamandosi all'eliminazione di tutti quei concetti presupposti nella comprensione del mondo atomico e sub-atomico che non avevano un corrispettivo sperimentale-operativo, come i concetti di spazio, tempo e moto assoluti aboliti dalla relatività. La nuova fisica di Heisenberg imponeva non solo l'abbandono della geometria delle traiettorie, ma anche dell'aritmetica e dell'analisi, ovvero di tutta la matematica del continuo per le grandezze fisiche da sostituire con l'algebra non-commutativa delle matrici. Anche la più nota formulazione di Schrödinger implicava comunque una nuova algebra non-commutativa degli operatori. Come in relatività veniva spezzata la gerarchia fra geometria e fisi- 
ca e la crono-geometria quadridimensionale non-euclidea veniva determinata a posteriori, nella prospettiva quantistica veniva spezzata anche la gerarchia che subordinava la fisica all'aritmetica e all'algebra standard, e l'algebra non-commutativa veniva determinata sperimentalmente. Ma c'è di più: anche la logica classica veniva destituita di fondamento e così qualunque metafisica logica derivata da una metafisica teologica delle idee pure o del puro pensiero di pensiero. La logica diventava sperimentale, determinata dalla fisica sperimentale [4, cap. XV].

Con l'adozione di geometrie non-euclidee nella relatività speciale e generale - al di là del fatto se la geometria non-euclidea sia effettivamente la vera 'geometria della Natura' o un linguaggio più adeguato di quello della geometria euclidea per la rappresentazione dei fenomeni fisici crollava la gerarchia di costruzione della conoscenza scientifica che poneva la geometria come 'a priori' rispetto alla fisica: la geometria è 'a posteriori' rispetto alla fisica. Con l'adozione di algebre e aritmetiche 'non commutative' nella meccanica quantistica, allo stesso modo e al di là del valore 'ontologico' o epistemologico dato all'aritmetica e all'algebra, crollava la gerarchia di costruzione della conoscenza scientifica che poneva l'aritmetica e l'algebra come 'a priori' rispetto alla fisica: l'aritmetica e l'algebra sono 'a posteriori' rispetto alla fisica. E la stessa geometria e l'analisi sembrano perdere di senso per i processi microfisici, se non considerate ad un livello puramente probabilistico. Ma c'è di più: la meccanica quantistica implica anche l'adozione di una logica non-classica (non-aristotelica), e al minimo si deve scegliere una logica in cui non vale più la proprietà distributiva della congiunzione. Crollava così anche la gerarchia di costruzione della conoscenza scientifica che poneva la logica come 'a priori' rispetto alla fisica: anche la logica è 'a posteriori' rispetto alla fisica. E quanto si è detto prima implica certamente che anche l'epistemologia non possa più essere 'a priori' rispetto alla fisica: l'epistemologia non è il fondamento ultimo della conoscenza scientifica, non è la "scienza prima" che determina ciò che è reale o esperibile, ma è 'a posteriori' rispetto alla fisica e ai processi fisici.

La gnoseologia delle azioni sperimentali, in effetti, non può essere intesa come una teoria aprioristica della conoscenza: le stesse azioni sperimentali mostrano che la Natura non è mai determinabile in maniera completa e univoca e ogni conoscenza è probabilistica, gravata da incertezze ed errori.

Secondo la fisica quantistica, non si può definire neanche uno stato meccanico di moto, identificato da posizione e velocità, in quanto 
non esattamente definibili e misurabili congiuntamente secondo il "principio d'indeterminazione" formulato da Heisenberg nel 1927: la Natura non è rappresentabile secondo modelli meccanici, non è categorizzabile in termini delle grandezze fisiche meccaniche.

In meccanica quantistica, per il principio d'indeterminazione, è impossibile definire lo stato di moto (iniziale) e quindi è impossibile determinare e predire il moto, non per l'inesattezza di ogni misura sperimentale e per la conseguente ignoranza, ma per l'indefinibilità intrinseca delle grandezze fisiche (coniugate), coinvolte nella rappresentazione del moto, al di sotto di una certa indeterminazione. E' possibile scrivere un'equazione differenziale solo per le ampiezze di probabilità dell'esito delle misure: la conoscenza scientifica del mondo non è più univoca o certa, ma è intrinsecamente probabilistica. I fenomeni sono impredicibili e si possono stabilire solo delle regolarità statistiche. I fenomeni quantistici non sono raggruppabili in catene causali né in catene probabilistiche: sono intrinsecamente acausali. Crolla il principio di causalità. Lo stesso metodo sperimentale non garantisce la ripetibilità senza condizioni delle misure e degli esperimenti, né può fornire le basi di un 'determinismo meccanico-tecnico', ma al contrario le misure sono correlate ad un'intrinseca indeterminazione dei processi fisici e ad una loro conoscenza mai certa ed univoca. Cambia il concetto di scienza, che perde la sua caratterizzazione di predicibilità, legato allo stabilimento di una conoscenza certa e univoca. Il "linguaggio" matematico, come anche il metodo sperimentale, in effetti non può assolvere questa funzione di accesso ad una conoscenza certa e univoca della realtà.

Crollano così le stesse basi metodologiche ed epistemologiche che caratterizzavano la scienza moderna fra Seicento e Settecento in opposizione alla precedente caratterizzazione della Natura, della scienza e dell'epistemologia.

Ma c'è di più. Il continuismo storico delle teorie fisiche non permette di comprendere che la stessa meccanica quantistica è stata falsificata e sostituita dalla teoria quanto-relativistica di campo.

La prospettiva quanto-relativistica di campo con la seconda quantizzazione, come compreso da Landau e Peierls già nel 1930-1931 [8] [9][10], comporta nuove più radicali indeterminazioni che decostruiscono il concetto di corpuscolo materiale, e, come notato da Giuliano Preparata, la complementarità onda-corpuscolo [11].

Il concetto di simultaneità usato nella formulazione del principio d'indeterminazione è relativisticamente un assoluto di cui doversi disfa- 
re: lo spazio-tempo in cui non si possono fare misure di entrambe le due variabili coniugate si estende a tutta la cosiddetta zona di sincronicità d'intervalli spazio-temporali spacelike. Ma questa, per il principio d'indeterminazione stesso, non è più distinguibile dalla zona di causalità d'intervalli spazio-temporali timelike. L'indeterminazione fa sì che causalità e sincronicità non si distinguano più e si ha così la possibilità di comprendere il fenomeno dell'entanglement.

Ma non è tutto: non risulta più possibile fare misure di quantità di moto e di energia in un istante o in tempi brevi, la loro esattezza richiederebbe un tempo infinito (per la quantità di moto l'indeterminazione è circa $\hbar / c$ per l'inverso dell'indeterminazione sul tempo, $\Delta p \Delta t \sim$ $\hbar / c$; l'errore sulla posizione di una particella può arrivare a coincidere con la lunghezza d'onda di De Broglie, $\Delta x \sim \hbar /(m c)$ o $\hbar / p$, che implica che il fotone non sia mai localizzabile. Tutto questo comporta che la funzione d'onda di una certa grandezza ad un istante non sia definibile, non corrispondendo ad un'informazione osservabile, fisicamente disponibile. L'indeterminazione su quantità di moto ed energia corrispondono a quelle di creazione-distruzione di una coppia di particellaantiparticella $\left(\Delta p \sim m c\right.$ e $\left.\Delta E \sim m c^{2}\right)$, che significa che crolla la possibilità di definire una particella come un corpuscolo materiale isolato: l'indeterminazione cioè corrisponde a un mutamento reale (ciò che è virtuale ha solo un tempo d'esistenza più breve), non si tratta di semplice ignoranza sul piano epistemologico; tutto ciò implica che la particella debba essere considerata come particolare eccitazione dello stato di vuoto di un campo (gli effetti Casimir, ancora di più quello dinamico, e Lamb mostrano il "vuoto" come sorgente e "specchio" di tutte le particelle possibili virtuali) [12][13].

La prospettiva quanto-relativistica di campo mostra come la probabilità legata alla funzione d'onda non possa essere interpretata in senso soggettivo e che la sua interpretazione debba essere tutt'uno con il fatto che la particella costituisca il quanto di un campo fisico effettivo: il campo propaga anche informazione sulla stessa presenza o assenza di una particella in una zona di spazio-tempo sempre finita nella sua indeterminazione; questo implica che l'informazione sia una grandezza fisica oggettiva che costituisce la stessa materializzabilità del campo in un quanto-particella. I quanti-particelle non esistono come entità separate, localizzabili e indipendenti, ma sono solo legate alle misure locali nello spazio-tempo del campo. Il fenomeno dell'entanglement mostra che, come parti di campi, i quanti/particelle sono sempre correlati da inter- 
valli spazio-temporali nulli e non spacelike o timelike, e quindi l'informazione legata a un quanto non è indipendente dal resto dei quanti e dai campi nella loro intera totalità. Una particella materiale è nient'altro che un'informazione parziale e incompleta del campo; un sistema di due particelle legate in un qualche passato è in realtà il sistema di un campo e questo campo non è fattorizzabile nel prodotto di due campi separabili.

La teoria quanto-relativistica di campo standard può definire $\mathrm{i}$ campi solo nel limite di non interazione dello stato iniziale e finale (condizione di libertà asintotica dei campi) di un processo di transizione: per questo si può misurare effettivamente solo la matrice $S$ di scattering, il cui modulo quadro dà la probabilità del processo.

Al di là della teoria della cosidetta "democrazia nucleare", la realtà dinamica d'interazione dell'universo è descrivibile solo da un campo a infinite componenti di Majorana non fattorizzabile [14][15]. L'individualità particellare-corpuscolare-materiale appare così come un presupposto metafisico falsificato. L'unità dell'universo non è solo ed eventualmente sostanziale, ma dinamica effettiva: costituisce così, a livello profondo e non direttamente esperibile, l'equivalente di una totalità organizzata e vivente, la Natura come la Physis della pre-comprensione arcaica e antica pre-socratica.

La prospettiva quanto-relativistica dello spazio-tempo gravitazionale implica poi il dover prendere in considerazione tutte le possibili geometrie e corrispondentemente tutte le possibili logiche [16]. La fisica quantistica non esprime più un logos umano aprioristico, ma piuttosto un logos proprio della physis ma solo a posteriori conoscibile e mai completamente e determinatamente. Seppure l'indeterminazione non può essere considerata in termini di libertà, ne costituisce però la condizione di possibilità, della libertà in senso esistenziale: la fisica quantorelativistica supera così l'antico problema dell'auto-comprensione fenomenologico-esistenziale della libertà e della responsabilità etica umana all'interno della Natura, esclusa dall'antico fatalismo come dalla teologia della predistazione e dalla precedente fisica determinista.

Bisogna quindi ripensare la Natura: la Quantum-Relativistic Physis costituisce una realtà del tutto indeterminata di un campo totale a infinite componenti non fattorizzabili in parti, che va al di là di una qualsiasi forma di realismo speculativo-teoretico-metafisico (materialistico, locale, deterministico) riduttivo della realtà fisica a una rappresentazione razionale umana, che la sperimentazione contraddice. 


\section{BIBLIOGRAFIA}

[1] M. Heidegger (1952), Was heisst Denken?, tr. it., Cosa significa pensare?, in M. HeIDEgGer, Vorträge und Aufsätze, Neske, Pfullingen 1954; tr. it. di G. Vattimo, Cosa significa pensare?, in M. Heidegger, Saggi e discorsi, Mursia, Milano 1976, pp. 85-95.

[2] M. HeidegGeR (1951-52), Was heisst Denken?, Niemeyer, Tübingen 1954; tr. it. a cura di G. VATtimo, Che cosa significa pensare?, Sugarco, Milano 1978.

[3] H. Blumenberg, Die Legitimität der Neuzeit, Suhrkamp Verlag, Frankfurt am Main 1966, $1974^{2}$, tr. it. di C. Marelli, La legittimità dell'età moderna, Marietti, Genova 1992.

[4] E. GiannetTo, Un fisico delle origini. Heidegger, la scienza e la Natura, Donzelli, Roma 2010.

[5] E. Giannetto, Dispense di filosofia contemporanea, Università di Bergamo 2015-2016.

[6] M. HeIDEgger (1935), Die Frage nach dem Ding. Zu Kants Lebre von den transzendentalen Grundsätzen, Niemeyer, Tübingen 1962; tr. it. a cura di V. Vitiello, La questione della cosa, Guida, Napoli 1989.

[7] E. Giannetto, Henri Poincaré and the rise of special relativity, in Hadronic Journal Supplement 10 (1995), 365-433.

[8] L. LANDAU, Zum spin-Effekt im Mebrkörperproblem, Physikalische Zeitschrift 30 (1929) pp. 654-655.

[9] L. LANDAU \& R. PEIERLS, Erweiterung des Unbestimmtheitsprinzips für die relativistische Quantentheorie, Zeitschrift für Physik 69 (1931), pp. 56-69; tr. ingl. in D.Ter HAAR, ed.:Collected Papers of Landau (Pergamon Press, Oxford, 1965), pp. 40-51, e anche in J. A. WHeELER \& W. H. ZureK, eds., Quantum Theory and Measurement (Princeton University Press, Princeton, 1983), pp. 465-476.

[10] V.B. Berestetskit, E.M. LifShitz, L.P. PitAevskiI, Relativistic Quantum Theory, Pergamon Press, Oxford, 1-4 (1971), Vol. 4 of L. LANDAU \& E.M. LifshitZ, Course of Theoretical Physics.

[11] G. Preparata, An Introduction to a Realistic Quantum Physics, World Scientific, Singapore 2002.

[12] Pasi Lähteenmäki, G. S. Paraoanu, Juha Hassel, and Pertti J. Hakonen, Dynamical Casimir effect in a Josephson metamaterial, in Proceedings of the National Academy of USA, vol. 110 no. 11 (2013), 4234-4238.

[13] Willis E. Lamb, Robert C. Retherford, Fine Structure of the Hydrogen Atom by a Microwave Method, in Physical Review 72 (1947), p. 241.

[14] E. MAJORANA, Teoria relativistica di particelle con momento intrinseco arbitrario, in Nuovo Cimento 9 (1932), pp. 335-344; ristampato in E. Amaldi, La vita e le opere di Ettore Majorana, Accademia dei Lincei, Roma, 1966.

[15] E. GiannetTo, Su alcuni manoscritti inediti di E.Majorana, in F. Bevilacqua, a cura di, Atti del IX Congresso Nazionale di Storia della Fisica, Urbino 1988, Goliardica Pavese, Pavia, 1989, pp. 173-185.

[16] E. GiannetTo, Toward a Physical Superlogodynamics, in Logic Colloquium '88. Padova, in Journal of Symbolic Logic 55 (1990) 404-405. 